\title{
Adult Respiratory Distress Syndrome in Patients with Malignant Astrocytoma
}

\author{
-Three Case Reports-
}

\author{
Toshihiro KumaBe, Teiji TominAGa, Takashi Yoshimoto, \\ and Takamasa KAYAMA* \\ Department of Neurosurgery, Tohoku University School of Medicine, Sendai; ${ }^{*}$ Department of \\ Neurosurgery, Yamagata University School of Medicine, Yamagata
}

\begin{abstract}
Three patients treated for intracranial malignant astrocytomas developed adult respiratory distress syndrome (ARDS). All three patients had a Karnofsky performance status score of at least $80 \%$ and similar clinical characteristics. All demonstrated hypersensitivity to phenytoin. ARDS occurred in two patients during radiotherapy and in one with radiation-induced brain damage following initial radiochemotherapy. ARDS occurred shortly after tapering the long-term administration of betamethasone. The initial symptoms were dry cough, fever, and generalized toxic eruption. Serum lactic acid dehydrogenase ( $\mathrm{LDH}$ ) concentrations were increased up to 2500 IU/l. Several days later, the patients suddenly complained of dyspnea. All patients fulfilled the diagnostic criteria for ARDS. Two patients recovered, but a young woman died. Clinical symptoms such as fever of unknown origin, dry cough, and skin eruptions, accompanied by abnormally increased serum LDH concentrations during or following the tapering of long-term administration of corticosteroids, are warning signs of ARDS in patients being treated for malignant gliomas.
\end{abstract}

Key words: adult respiratory distress syndrome, cytokine, glioma, phenytoin, steroids

\section{Introduction}

Adult respiratory distress syndrome (ARDS) was first described in $1967 .{ }^{11}$ ARDS occurs as a consequence of the lung's response to a wide variety of injuries to its capillary endothelium and alveolar epithelium. ${ }^{3,13)}$ ARDS is not a disease but rather a disorder that represents the final common pathway of the lung's pathophysiological responses to many kinds of injuries. ${ }^{10)}$ The American-European Consensus Committee on ARDS proposed that the term "acute lung injury" should be applied to a wide spectrum of entities, and that ARDS should be reserved for conditions representing the most severe end of this spectrum. ${ }^{33}$

ARDS is characterized by diffuse pulmonary microvascular injury with an almost uniform pattern of roentgenographical and physiological abnormalities: diffuse alveolar infiltrations caused by in- creased capillary permeability, pulmonary edema, and severe hypoxemia caused by intrapulmonary shunting of blood, and marked decreases in lung compliance and functional residual capacity caused by alveolar flooding and changes in surface tension. ${ }^{101}$ The mortality rate in patients with ARDS varies from $10 \%$ to $90 \%$, depending on the diagnostic criteria. The recommended diagnostic criteria for ARDS include: acute onset, $\mathrm{PaO}_{2} / \mathrm{FiO}_{2}<200 \mathrm{mmHg}$ regardless of positive end-expiratory pressure (PEEP), bilateral infiltrates observed on a frontal chest radiograph, and pulmonary artery wedge pressure (PAWP) $<18 \mathrm{mmHg}$ or no clinical evidence of left atrial hypertension. ${ }^{3)}$ However, this mortality rate has not improved since ARDS was first described.

We describe three patients who developed ARDS among 34 patients treated for malignant astrocytoma within a 20 -month interval.

Received September 4, 1996; Accepted March 4, 1997 

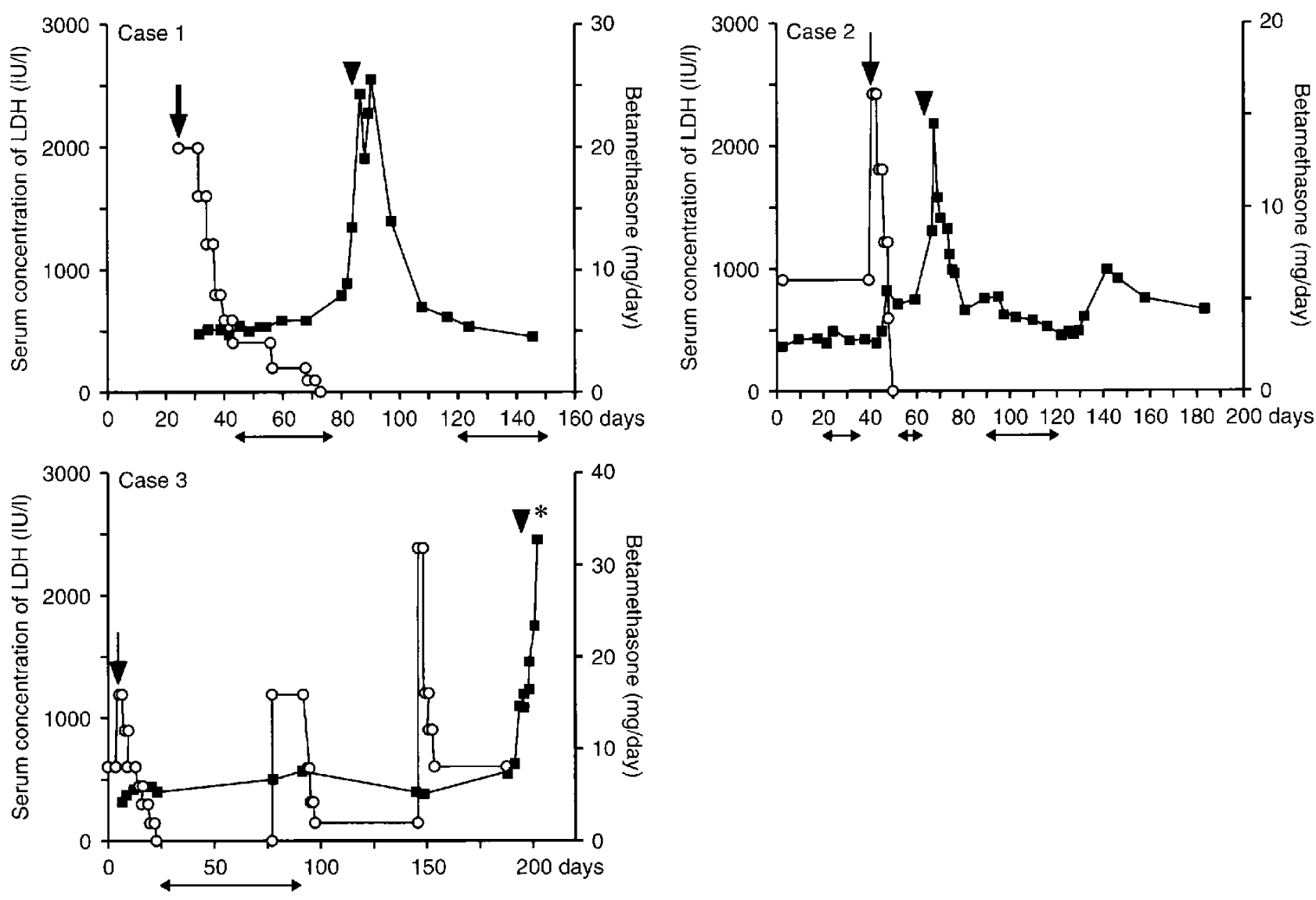

Fig. 1 Clinical course of three patients with adult respiratory distress syndrome (arrowhead). $\square$ : serum lactic acid dehydrogen ase $(\mathrm{LDH})$ concentration, $\bigcirc$ : betamethasone, double-headed arrow: radiation, arrow: resection, thin arrow: resection + intraoperative radiation, asterisk: death.

\section{Case Reports}

The clinical courses for the three patients are summarized in Fig. 1.

Case 1: A 45-year-old female was admitted with temporal lobe epilepsy in September 1992. There was no history of previous illness. Neurological examination revealed only left upper homonymous quadrantanopsia. Magnetic resonance (MR) imaging revealed a right temporal intrinsic tumor with slight enhancement. She was given phenytoin $(250 \mathrm{mg}$ daily) to control her seizures. Two weeks after starting phenytoin medication, she had fever, and developed a morbilliform rash on her chest, trunk, and extremities. Phenytoin medication was discontinued, and zonisamide $[300 \mathrm{mg}$ daily] was substituted. The symptoms disappeared in 2 weeks. The tumor was partially resected through a right temporal craniotomy in October 1992. The histological diagnosis was anaplastic astrocytoma. She was treated with betamethasone (starting with $20 \mathrm{mg}$ daily, then ta- pered), femotidine ( $40 \mathrm{mg}$ ), zonisamide ( $300 \mathrm{mg}$ ), and chemotherapy with 1-(4-amino-2-methyl-5-pyrimidinyl)methyl-3-(2-chloroethyl)-3-nitrosourea hydrochloride (ACNU). She received fractionated radiotherapy at 2 Gy per day, 5 days per week, delivered to the local brain through parallel opposed ports with megavoltage equipment. Her condition was stable, and her Karnofsky performance status score ${ }^{12)}$ was $90 \%$.

Five days after betamethasone medication was discontinued, she began to complain of dry cough and fever, and developed a generalized rash. Her serum lactic acid dehydrogenase (LDH) level increased gradually. The rash progressed, despite immediate discontinuation of all medication. After another 5 days, she suddenly complained of dyspnea. Chest roentgenography revealed bilateral diffuse infiltrates. Her $\mathrm{PaO}_{2}$ breathing room air was decreased to 44 torr. She required endotracheal intubation and mechanical ventilation with PEEP. The PAWP was measured as under $18 \mathrm{mmHg}$. Her serum LDH level 
increased up to $2535 \mathrm{IU} / \mathrm{l}$ (normal 198-424 IU/1). Endotracheal aspirate contained no pus, and culture identified no known pathogens, except Candida albicans. Methylprednisolone (1 $\mathrm{g}$ per day) was administered intravenously for 3 days. She also received antibiotics and antimycotic agents. Fortunately, her condition improved, and she was extubated 7 days after intubation. Her serum LDH level began to decrease, and normalized after 1 month. Femotidine and zonisamide were prescribed after this incidence without any problems. She received additional irradiation of the tumor with a total dose of $67 \mathrm{~Gy}$, and then was discharged home.

Case 2: A 63-year-old female was admitted with complaints of headache in December 1993. There was no history of previous illness other than hypercholesterolemia treated with 3-hydroxy-3-methylglutaryl-coenzyme A (HMG-CoA) reductase. Following admission, she was treated with phenytoin $(250$ mg daily) for seizure prophylaxis, betamethasone (6 $\mathrm{mg}$ daily), and femotidine (40 $\mathrm{mg}$ daily). MR imaging revealed a right temporal intrinsic tumor with marked enhancement. She underwent irradiation of the tumor with a total dose of $20 \mathrm{~Gy}$. Two weeks after starting the phenytoin medication, she had fever, and developed a morbilliform rash on her chest, trunk, and extremities. Phenytoin was replaced by zonisamide (200 $\mathrm{mg}$ daily), and the symptoms resolved within 2 weeks. MR imaging after the radiotherapy demonstrated a marked decrease in tumor size and enhancement. The tumor was subtotally resected through a right temporal craniotomy and intraoperative radiation ( $15 \mathrm{~Gy}$ ) was performed in January 1994. The histological diagnosis was glioblastoma multiforme. Postoperatively, femotidine and $16 \mathrm{mg}$ of betamethasone a day was administered intravenously, this medication was then tapered and finally discontinued on the 7th postoperative day. Her Karnofsky performance status score was $\mathbf{8 0} \%$. Subsequently, fractionated radiotherapy was begun to the local brain.

Ten days after the operation, she developed a generalized morbilliform rash, and her serum LDH level gradually increased. HMG-CoA reductase and zonisamide medication was discontinued. She was also infected with oral Herpes simplex virus, which was successfully treated with aciclovir. Seven days later, in mid-February, she began to complain of dry cough and fever. After another 4 days, she complained of acute dyspnea, and chest roentgenography revealed bilateral diffuse infiltrates (Fig. 2). Her $\mathrm{PaO}_{2}$ breathing room air was decreased to 38 torr. She underwent emergent endotracheal intubation and mechanical ventilation with PEEP. The PAWP was measured as under $18 \mathrm{mmHg}$. Her serum
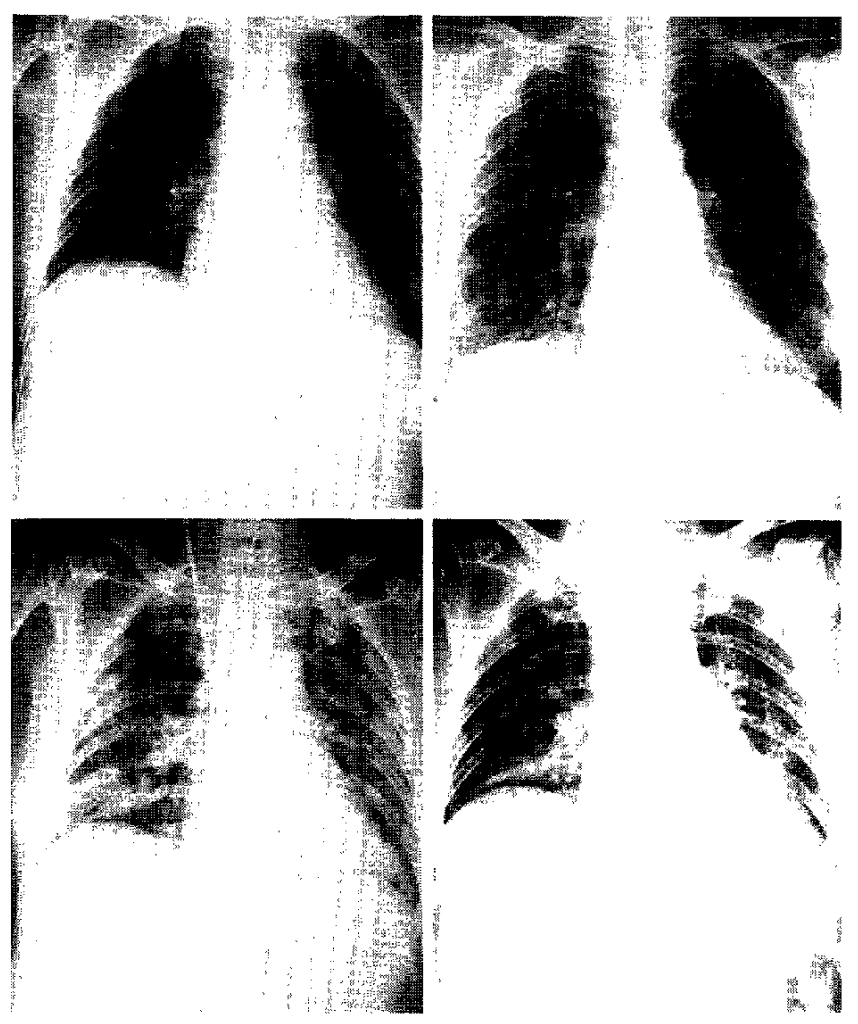

Fig. 2 Case 2. Serial chest roentgenograms, on admission showing no pathological findings (upper left), at onset of acute dyspnea showing only minimal and scattered interstitial infiltrates (upper right), during endotracheal intubation and mechanical ventilation showing diffuse, extensive bilateral interstitial and alveolar infiltrates (lower left), and just after the extubation showing clearing of diffuse bilateral infiltrates (lower right).

LDH level had increased to $2175 \mathrm{IU} / \mathrm{l}$. Endotracheal aspirate contained no pus, and culture identified no known pathogens except Candida albicans. Methylprednisolone ( $1 \mathrm{~g}$ per day) was administered intravenously for 3 days, followed by $60 \mathrm{mg}$ of predonine a day with femotidine. She also received antibiotics and antimycotic agents. Fortunately, her condition improved, and she was extubated 9 days following intubation. Her serum LDH level decreased, and normalized within 1 month. The dosage of predonine was tapered, and then maintained at $20 \mathrm{mg}$ a day with femotidine. She received additional irradiation of the tumor with a total dose of $63 \mathrm{~Gy}$. She was discharged home in May 1994.

Case 3: A 36-year-old female was admitted with severe headache and nausea in April 1994. There was no history of previous illness. On admission, she had 

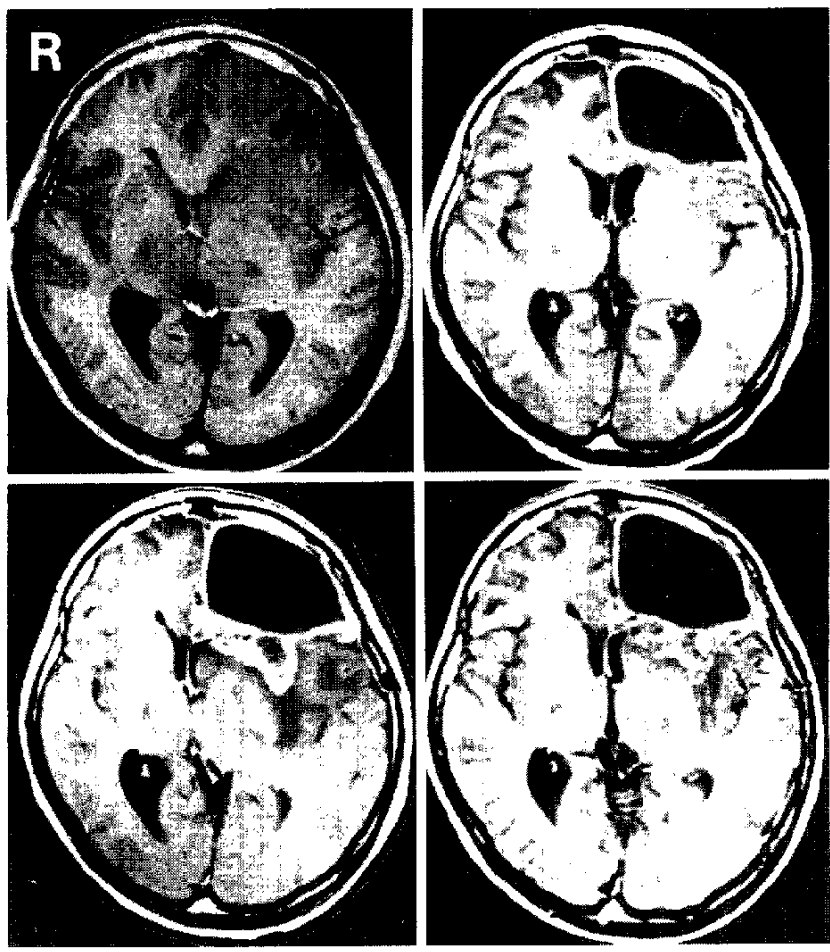

Fig. 3 Case 3. Serial axial gadolinium-enhanced $\mathrm{T}_{1}$-weighted magnetic resonance images, on first admission demonstrating a left frontal infiltrative intrinsic tumor with slight enhancement effect (upper left), after completing initial treatment demonstrating only minimal enhanced lesion around the excised area (upper right), on second admission demonstrating enlargement of the enhanced lesion and edema (Iower left), and after high-dose administration of betamethasone demonstrating reduction of the enhanced lesion and edema (lower right).

no neurological deficits except bilateral papilledema. MR imaging revealed a left frontal infiltrative intrinsic tumor with slight enhancement (Fig. 3 upper left). She was given phenytoin (250 mg daily) for seizure prophylaxis, betamethasone ( $8 \mathrm{mg}$ daily), and femotidine ( $40 \mathrm{mg}$ daily). A left frontal lobectomy and $15 \mathrm{~Gy}$ of intraoperative radiation therapy were performed in April 1994. The histological diagnosis was anaplastic astrocytoma. Ten days after the operation, she had fever, and a generalized morbilliform rash. Phenytoin was discontinued, and changed to zonisamide ( $300 \mathrm{mg}$ daily). These symptoms disappeared in 2 weeks. After the operation, $16 \mathrm{mg}$ of betamethasone a day was administered intravenously with femotidine, tapered rapidly, and finally stopped on the 14th day after the operation. She received fractionated radiotherapy to the local brain
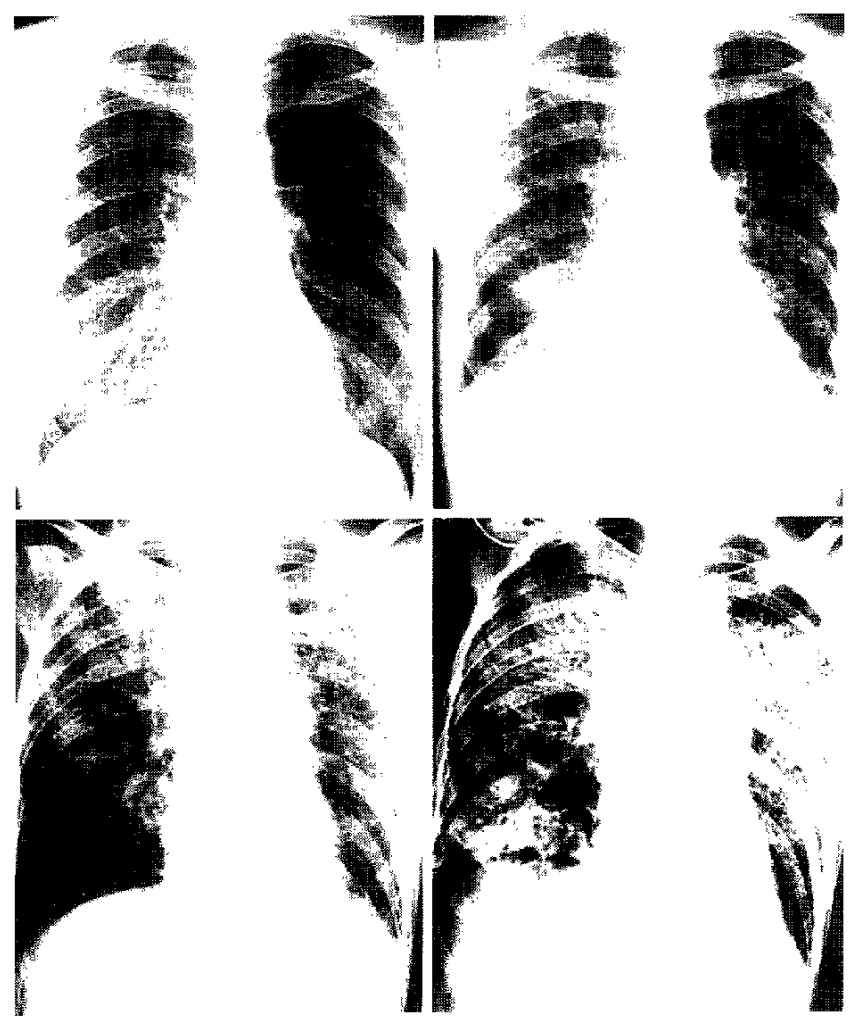

Fig. 4 Case 3. Serial chest roentgenograms, on first admission demonstrating no pathological findings (upper left), on the day she complained of dyspnea demonstrating scattered interstitial infiltrates (upper right), during endotracheal intubation and mechanical ventilation demonstrating diffuse, extensive bilateral interstitial and alveolar infiltrates when barotrauma such as pneumothorax was confirmed (lower left), and a day before her death demonstrating worsening of bilateral diffuse infiltrates (lower right).

with a total dose of $63 \mathrm{~Gy}$, and chemotherapy with ACNU. During the radiochemotherapy, MR imaging revealed enhancement and edema around the resected area. Thallium-201 single photon emission computed tomography $\left({ }^{201} \mathrm{Tl}\right.$ SPECT) showed some uptake into the enhanced area. We considered this to be radiation-induced brain damage with some tumor regrowth. Betamethasone (16 mg per day) with femotidine was administered. During this treatment, she suffered from Herpes zoster at her maxillar nerve region. This was successfully treated with aciclovir. MR imaging showed that the enhanced area and edema were decreased markedly 2 weeks later (Fig. 3 upper right). Betamethasone was tapered to $4 \mathrm{mg}$ a day and maintained. She was discharged 
home in July 1994.

She began to complain of severe headache again at the end of August. MR imaging revealed enlarge. ment of the enhanced lesion and edema (Fig. 3 lower left). ${ }^{201} \mathrm{Tl}$ SPECT showed marked uptake into this enhanced area. She was readmitted on September 6 , 1994. Betamethasone (32 mg per day) was administered intravenously for 3 days, and then tapered. MR imaging on September 14 revealed reduction of the lesion (Fig. 3 lower right). She was observed as an outpatient. About a month after the second discharge (betamethasone had been reduced to $8 \mathrm{mg}$ a day), she began to complain of dry cough and fever, and developed a generalized morbilliform rash. Her Karnofsky performance status score was $80 \%$ at that time. After another 7 days, she complained of dyspnea, and was readmitted. Her $\mathrm{PaO}_{2}$ breathing room air was decreased to 67.7 torr. Her serum LDH level was increasing gradually. Chest roentgenography revealed bilateral diffuse infiltrates (Fig. 4). We administered $1 \mathrm{~g}$ of methylprednisolone a day intravenously for 3 days. She also received antibiotics and antimycotic agents. However, her condition deteriorated progressively, and she required endotracheal intubation and mechanical ventilation with PEEP. Endotracheal aspirate contained no pus, and culture identified no known pathogens, except Candida albicans. Her condition deteriorated further, and she died in November 1994.

\section{Discussion}

These three patients demonstrated similar clinical courses. All had a high performance status before suffering from ARDS. None had known risk factors for the development of ARDS, such as aspiration of gastric contents, burns, cardiopulmonary bypass, disseminated intravascular coagulation, multiple fractures, multiple transfusions, near-drowning, pancreatitis, prolonged hypotension, sepsis, toxic inhalation, or trauma. ${ }^{13}$ Their courses prior to the onset of ARDS shared the following characteristics: hypersensitivity to phenytoin; symptoms including fever of unknown origin, dry cough, and a generalized rash; tapering of long-term administration of betamethasone; and increased serum concentrations of $\mathrm{LDH}$.

The pathogenesis of ARDS may involve two pathways: the direct effects of an insult on lung cells and the indirect result of an acute systemic inflammatory response. ${ }^{3)}$ Free radicals, proteinases, and soluble agents including cytokines, arachidonic acid metabolites, and charged proteins are possible mediators of lung injury in ARDS. ${ }^{8]}$ ARDS may be caused by excessive, uncontrolled activation of endogenous inflammatory cells and mediators. Circulating inflammatory cytokines, such as tumor necrosis factor- $\alpha$ (TNF- $\alpha),{ }^{2,7,11)}$ interleukin- $1 \beta$, and interleukin-6, may be involved in the development of ARDS in patients with a history of severe blunt trauma, hemorrhagic shock, and major vascular surgery. ${ }^{16)}$

The exact mechanism of development of ARDS in these patients could not be identified. The possibility of virus infection, such as cytomegalovirus, could not be ruled out. All three patients were immunocompromised due to steroid therapy and the syndrome developed as the steroid medication was tapered off, raising the possibility that they were beginning to respond to a pre-existing infection. All three patients were treated with antibiotics, so even the possibility of bacterial pneumonia existed.

Two patients demonstrated skin reaction to phenytoin during radiation therapy, and one did shortly after the surgical intervention with intraoperative radiation therapy. Skin reactions occur in 2$10 \%$ of all patients receiving phenytoin, ${ }^{9,15]}$ so hypersensitivity in all three patients might not be accidental. Severe cutaneous reactions, such as erythema multiforme or erythema multiforme bullosa (Stevens-Johnson syndrome), have occurred following the combination of phenytoin and brain radiation therapy. ${ }^{91}$ The combination of phenytoin, brain radiation therapy, and the tapering of steroids seem to cause a predisposal to erythema multiforme. However, the exact mechanism of phenytoin hypersensitivity is not known, and this clinical feature may also have been related to the development of ARDS. All three patients had also showed abnormal increases in serum LDH concentration before they complained of dyspnea. The serum LDH concentration decreased to the normal range within a month in the two patients who recovered. LDH 3 may be useful in determining the diagnosis in patients with ARDS. ${ }^{6)}$ The increase in the serum $L D H$ concentrations may also provide prognostic information.

The mainstay therapy for ARDS is management of the underlying etiological disorder, but this is not always possible. Assisted mechanical ventilation should be instituted in any patient with ARDS who suffers respiratory failure or impending respiratory failure. PEEP is the most effective way to maintain adequate oxygenation in patients with ARDS. There are no specific pharmacological agents of proven value for the treatment of ARDS. Corticosteroids and prostaglandin $\mathrm{E}_{1}$ have been widely used clinically, but no benefit in outcome, lung compliance, pulmonary shunting, chest radiography, severity score, or survival has been found. ${ }^{4,13)}$ Administration of 
steroids may provide benefit in some patients, but the role of corticosteroids in ARDS with so many different causes remains ambiguous. ${ }^{10)}$ The production of inflammatory cytokines can be controlled with corticosteroids, ${ }^{5}$ so some cases of ARDS, such as those caused by high serum concentrations of inflammatory cytokines, can be treated with steroids. Recently, pentoxifylline, which has a direct inhibitory effect on TNF- $\alpha$ secretion, has been assessed for the control of serum TNF- $\alpha$ concentrations in patients with cancer. ${ }^{14)}$ Modulation of inflammatory cytokine secretion might be effective for the prevention of ARDS.

Patients with gliomas, even those with a high performance status, can develop ARDS. Predictive clinical symptoms, such as fever of unknown origin, dry cough, and skin eruptions, are accompanied by abnormally increasing serum LDH concentrations during or after the tapering of long-term administration of corticosteroids.

\section{References}

1) Ashbaugh DG, Bigelow DB, Petty TL, Levine BE: Acute respiratory distress in adults. Lancet 2: 319323,1967

2) Balkwill F, Burke F, Talbot D, Tavernier J, Osborne R, Naylor S, Durbin H, Fiers W: Evidence for tumour necrosis factor/cachectin production in cancer. Lancet 2: 1229-1231, 1987

3) Bernard GR, Artigas A, Brigham KL, Carlet J, Falke K, Hudson L, Lamy M, LeGall JR, Morris A, Spragg R: The Consensus Committee: Report of the American-European consensus conference on ARDS: Definition, mechanisms, relevant outcomes and clinical trial coordination. Intensive Care Med 20: 225-232, 1994

4) Bernard GR, Luce JM, Sprung CL, Rinaldo JE, Tate RM, Sibbald WJ, Kariman K, Higgins S, Bradley R, Metz CA, Harris TR, Brigham KL: High-dose corticosteroids in patients with the adult respiratory distress syndrome. N Engl J Med 317: 1565-1570, 1987

5) Beutler B, Krochin N, Milsark IW, Luedke C, Cerami A: Control of cachectin (tumor necrosis factor) synthesis: Mechanism of endotoxin resistance. Science 232: 977-980, 1986

6] Chen ZT, Mao BL, Gou J: The role of oxygen radicals in adult respiratory distress syndrome. Chung Hua Nei Ko Tsa Chih 32: 594-596, 1993

7) Chiang C, McBride WH: Radiation enhances tumor necrosis factor- $\alpha$ production by murine brain cells. Brain Res 566: 265-269, 1991

8) Dal Nogare AR: Adult respiratory distress syndrome. Am J Med Sci 298: 413-430, 1989

9) Delattre JY, Safai B, Posner JB: Erythema multiforme and Stevens-Johnson syndrome in patients receiving cranial irradiation and phenytoin. Neurology 38: 194-198, 1988

10) Flick MR, Murray JF: High-dose corticosteroid therapy in the adult respiratory distress syndrome. JAMA 251: 1054-1056, 1984

11) Hallahan DE, Spriggs DR, Beckett MA, Kufe DW, Weichselbaum RR: Increased tumor necrosis factor$\alpha$ mRNA after cellular exposure to ionizing radiation. Proc Natl Acad Sci U S A 86: 10104-10107, 1989

12) Karnofsky DA, Abelmann WH, Craver LS, Burchenal $\mathrm{JH}$ : The use of nitrogen mustards in the palliative treatment of carcinoma: With particular reference to bronchogenic carcinoma. Cancer 1: 634-656, 1948

13) Lechin AE, Varon J: Adult respiratory distress syndrome (ARDS): The basics. J Emerg Med 12: 63-68, 1994

14) Lissoni P, Ardizzoia A, Perego MS, Grassi MG, Arosio M, D'Amico P, Cazzaniga M, Crispino S, Tancini G, Barni S: Inhibition of tumor necrosis factor-alpha secretion by pentoxifylline in advanced cancer patients with abnormally high blood levels of tumor necrosis factor-alpha. J Biol Regul Homeost Agents 7: 73-75, 1993

15) Pelekanos J, Camfield P, Camfield C, Gordon K: Allergic rash due to antiepileptic drugs: Clinical features and management. Epilepsia 32: 554-559, 1991

16) Roumen RMH, Hendriks T, van der Ven-Jongekrijg J, Nieuwenhuijzen GAP, Sauerwein RW, van der Meer JWM, Goris RJA: Cytokine patterns in patients after major vascular surgery, hemorrhagic shock, and severe blunt trauma. Relation with subsequent adult respiratory distress syndrome and multiple organ failure. Ann Surg 218: 769-776, 1993

Address reprint requests to: T. Kumabe, M.D., Department of Neurosurgery, Tohoku University School of Medicine, 1-1 Seiryo-machi, Aoba-ku, Sendai 980-77, Japan. 